\title{
Principal Component Analysis for Bacterial Proteomic Analysis
}

\author{
Y-h. Taguchi ${ }^{1, \star}$ and Akira Okamoto ${ }^{2}$ \\ 1 Department of Physics, Chuo University, 1-13-27 Kasuga, Bunkyo-ku, \\ Tokyo 112-8551, Japan \\ 2 Department of School Nursing and Health, Aichi University of Education, \\ 1 Hirosawa, Igaya-cho, Kariya, Aichi 448-8542, Japan
}

\begin{abstract}
Proteomic analysis is a very useful procedure to understand the bacterial behavioural responses to the external environmental factors. This is because bacterial genome information is mainly devoted to code enzyme for the control of the cellular metabolic networks. In this paper, we have performed proteomic analysis of Streptococcus pyogenes, which is known to be flesh-eating bacteria and can cause several human life-threatening diseases. Its proteome during growth phase is measured for four time points under two different culture conditions; with or without shaking. Its purpose is to understand the adaptivity to oxidative stresses. Principal component analysis is applied and turns out to be useful to depict biologically important proteins for both supernatant and cell components.
\end{abstract}

Keywords: Streptococcus pyogenes, proteomic analysis, principal component analysis.

\section{Introduction}

Streptococcus pyogenes is an important pathogen. The estimated annual number of Streptococcus pyogenes infection cases are more than 700 million. There are over 650,000 cases of severe, invasive infections that have a mortality rate of 25 $\%$. Although S. pyogenes is a normal bacteria flora, occasionally S. pyogenes can also cause life-threatening diseases. This means, it will be important to know what triggers the diseases that $S$. pyogenes causes. There are a huge number of researches [2] that investigate transcrptome responses to external environmental factor, but there are very few researches on how its proteome changes in response to external stimulations.

In this paper, we have systematically compared proteome of S. pyogenes during growing phases under two distinct culture conditions; with or without shaking. The latter condition was designed to be more oxidative stress condition. The purpose of this research is to know the proteomic response to these two different growth conditions. Using the principal component analysis (PCA) [12, we have selected representative proteins. Many of the representative proteins play biological roles during the incubation.

\footnotetext{
* Corresponding author. 


\section{Material and Methods}

\subsection{Proteome Analysis}

In this study, Streptococcus pyogenes (serotype M1) SF370 of a clinical isolate was investigated. The sample was incubated at $37{ }^{\circ} \mathrm{C}$ for $4,6,14$ and 20 hours $\left(O D_{660}=0.40,0.83,0.92\right.$, and 0.90, respectively $)$.

Bacterial cultures were separated into the supernatant and the cellular fractions by centrifugation. The reason why the cellular fraction was not divided into soluble/insoluble fractions in contrast to the previous researches 9 14] was because these two did not differ from each other so much in the preliminary investigations (not shown here). Proteins contained in each fraction were partially purified by ethanol-chloroform purification. After reduced alkylation, they were digested by Lysyl Endopeptidase and Trypsin and were provided as samples for mass spectrometry. Detection of digested proteins was performed by LTQ-Orbitrap XL (Thermo Fisher Sceintific Inc.). Spectrums obtained by LTQ were identified by MASCOT program combined with Paradigm MS4 LC system (Michrom BioResources Inc.), based upon the in-house amino acid database which consists of coding-sequence predicted by genomic analysis [4] and reevaluation of genome [10. To be identified, at least two unique amino acid sequences for each protein were required. False discovery rate was estimated by decoy databases constructed by randomized amino acid sequences. Each of two fractions was measured three times for each of four time points separately under two distinct culture conditions. Analyzed quantity by PCA was \%emPAI [5 13], which expresses the amount of proteins and \%emPAI was its normalized value. \%emPAI was normalized to have zero mean and unit variance before any analyses.

Hereafter, each sample was denoted by the tag ID in the form of XXXYY_Z, where XXX is either "sha" (the incubation under the shaking condition) or "sta" (the incubation under the static condition), YY denotes the duration time of the incubation $(05,07,14$, and 20 hours for the shaking incubation condition, and 04, 06, 14 and 20 hours for the static incubation condition), and Z is "wc" (the whole cellular fraction) or "snt" (the supernatant fraction), respectively.

\subsection{Transcriptome Data}

Transcriptome data set [1] with the accession number GSE5179 was downloaded from Gene Expression Omnibus (GEO). Raw data files GSM1167X.csv (X ranges from 67 to 79 ) were loaded into analysis program and column data named as F532.Median was used for further analyses. Each sample was normalized so as to have zero mean and unit variance. Then, six samples in the stationary phase were compared with six samples in the growth phase.

\subsection{Statistical Methods}

Application of Principal Component Analysis to Proteome Data. Suppose that we have proteome data $x_{s p}$, which is the normalized \%emPAI of $p$ th 
protein at $s$ th sample $(s=1, \ldots, S, p=1, \ldots, P)$. This data can be understood as two ways, i.e.,

Category 1. In total, there are supposed to be $S$ kinds of samples, each of which is characterized by the set of amounts of $P$ kinds of proteins; a set of $P$ dimensional vectors, the number of which is $S$.

Category 2. In total, there are supposed to be $P$ kinds of proteins, each of which is characterized by the amount of its expression at $S$ kinds of samples; a set of $S$ dimensional vectors, the number of which is $P$.

Principal component analysis (PCA) can be applied to both of the two cases. If PCA is applied to the former (Category 1), the $S$ kinds of samples are characterized with $D_{s}$ principal component scores $(\mathrm{PCSs}) y_{s}^{i},\left(i=1, \ldots, D_{s}\right)$, as

$$
\begin{aligned}
\mathbf{x}_{s} & =\left(y_{s}^{1}, y_{s}^{2}, \ldots, y_{s}^{D_{s}}\right) \\
y_{s}^{i} & =\sum_{p} a_{i p} x_{s p}
\end{aligned}
$$

instead of $P$ kinds of proteins. Alternatively, if PCA is applied to the later (Category 2), the $P$ kinds of proteins are characterized with $D_{p}$ PCSs $y_{p}^{i}$, $\left(i=1, \ldots, D_{p}\right)$, as

$$
\begin{aligned}
\mathbf{x}_{p} & =\left(y_{p}^{1}, y_{p}^{2}, \ldots, y_{p}^{D_{p}}\right) \\
y_{p}^{i} & =\sum_{s} a_{j s} x_{s p}
\end{aligned}
$$

instead of $S$ kinds of samples.

Selection of Representative Proteins. In some cases, PCA can be used to select representative $P^{\prime}(<P)$ proteins 914 as follows. At first, each protein is embedded into $D_{p}^{\prime}\left(<D_{p}\right)$ dimensional space (typically, $D_{p}^{\prime}$ is taken to be 2 ) by category $2 \mathrm{PCA}$. Then, the set $S_{p}$ of top $P^{\prime}$ proteins which are far from origin are decided, i.e.,

$$
S_{p} \equiv\left\{p \mid \operatorname{rank}_{p}\left[\sum_{i=1}^{D_{p}^{\prime}}\left(y_{p}^{i}\right)^{2}\right] \leq P^{\prime}\right\}
$$

where $\operatorname{rank}_{p}\left[f_{p}\right]$ is the descent rank order of the element $f_{p}$. For example, when $f_{2}<f_{3}<f_{1}<\cdots, \operatorname{rank}_{p}\left[f_{1}\right]=3, \operatorname{rank}_{p}\left[f_{2}\right]=1, \operatorname{and}_{P^{\prime}} \operatorname{rank}_{p}\left[f_{3}\right]=2$.

$P^{\prime}$ is decided to take a minimum number such that $y_{s}^{i},\left(i=1, \ldots, D_{s}^{\prime}<D_{s}\right)$, where typically $D_{s}^{\prime}$ is taken to be 2 , computed only with the selected $P^{\prime}$ proteins does not differ very much from the original $y_{s}^{i}$ computed with all proteins.

This procedure is repeated after removing $P^{\prime}$ proteins, i.e., PCA is applied to the remaining $P-P^{\prime}$ proteins. Then we get additional set $S_{p^{\prime}}$ of $P^{\prime \prime}\left(<P-P^{\prime}\right)$ proteins to express new PCSs obtained by $P-P^{\prime}$ proteins. 
$P$-Values to Describe the Difference of Transcriptome between the Growth Phase and the Static Phase. Using the two sided $t$-test, we get $P$-values to check if gene expression in each phase differs from each other. Then, the obtained $P$-values are attributed to each gene. After that, 1643 genes have significant $P$-values $(P<0.05)$ even after the application of FDR correction based upon BH criterion, among 1798 genes to which Spy-IDs are attributed.

\section{Results}

\subsection{Overview of Proteome with PCA Analysis}

Figure 11A shows two dimensional embedding of samples using the category 1 PCA. Then $P^{\prime}=23$ proteins (Table 1) are selected based upon the two dimensional embedding (not shown here) of proteins obtained by category 2 PCA. Hereafter we call this as round one selection. After that, all of samples are reembedded into two dimensional space (Fig. 1B) by category 1 PCA. Since Fig. $1 \mathrm{~B}$ is almost identical with Fig. 11A, configuration seen in Fig. 11A turns out to be dependent upon the selected $P^{\prime}$ proteins only.

Table 1. Round one representative proteins. Ribosomal proteins are underlined. The proteins in italic letter are mentioned in the text.

\begin{tabular}{|c|c|c|c|}
\hline SPy1489:hlpA & SPy2039 & & SPy2018:emm1 \\
\hline SPy0059:rpmC & SPy0611:tufA & SPy0062:rplX & SPy2043:mf \\
\hline Py061 & 9:AhpC SPy1831:rpsF & $\overline{\text { SPy2160:rpmG }}$ & SPy1373:ptsH \\
\hline SPy0731:eno & SPy1371:gapN $\overline{\text { SPy1881:pgk }}$ & SPy0711:speC & SPy0071:rpmD \\
\hline SPy2070:grol & SPy0712:mf2 & & \\
\hline
\end{tabular}

Above these procedures are repeated again for the remaining $P-P^{\prime}$ proteins and we have successfully selected round two representative proteins $P^{\prime \prime}=30$. (Figure 2 and Table 2).

Table 2. Round two representative proteins. Notations are the same as in Table 1.

\begin{tabular}{|c|c|c|c|c|}
\hline SPy0076:rpmJ & SPy1888:rpmE & SPy0063:rplE & SPy0717:rpmE & SPy1429:gpmA \\
\hline$\overline{\text { SPy0822:rpmA }}$ & SPy0273:fus & $\overline{\text { SPy2092:rpsB }}$ & $\overline{\text { SPy0051:rplW }}$ & SPy1282:pyk \\
\hline SPy0055:rplV & SPy1835:trx & SPy1889:fba & $\overline{\mathrm{SPy} 1294}$ & :arcB \\
\hline$\overline{\text { SPy085\%:mur1.2 }}$ & SPy0460:rplK & SPy0069:rpsE & SPy0272:rpsG & SPy1932:rplM \\
\hline SPy1261 & $\overline{\text { SPy1547:sagP }}$ & $\overline{\text { SPy1801:isp2 }}$ & $\overline{\text { SPy1262 }}$ & $\overline{S P y 1436: m f 3}$ \\
\hline SPy1234:rpsT & SPy0052:rplB & SPy2072:groE & SPy0913 & SPy1613 \\
\hline
\end{tabular}


A)

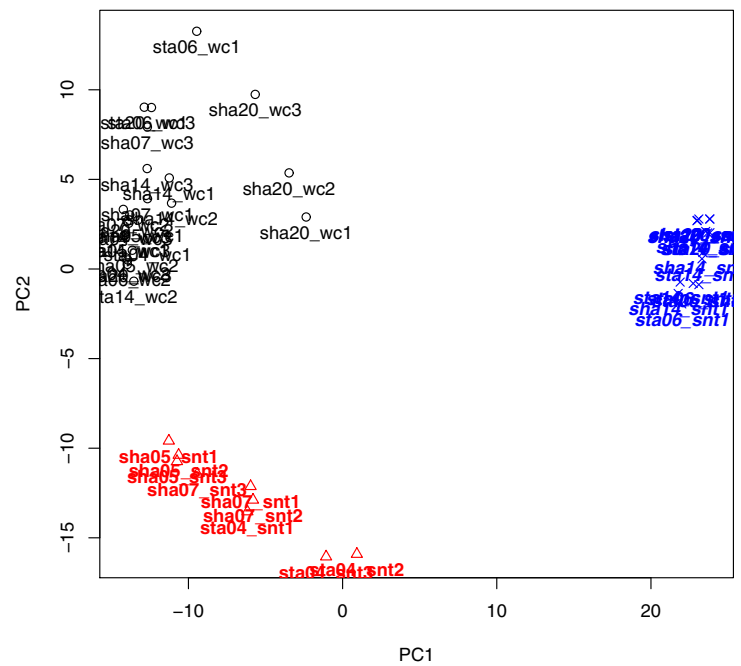

B)

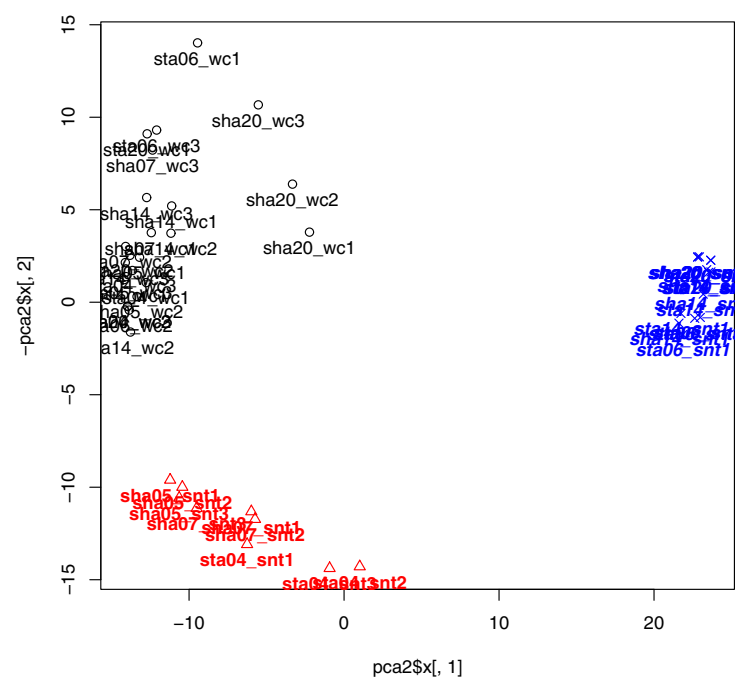

Fig. 1. A) Two dimensional embeddings of samples by Category 1 PCA. Black $(\bigcirc$, normal): the whole cellular experiments (wc experiments), Red ( $\triangle$, bold): the early phase extracellular proteomes (sha05_snt, sha07_snt, and sta04_snt experimets), and Blue $(\times$, bold italic): the late phase extracellular proteomes (sha14_snt, sha20_snt, sta06_snt, sta14_snt, and sta20_snt experiments) B) The same as A) but using only the selected $P^{\prime}=23$ proteins shown in Table 1. Cumurative contribution upto the second $\mathrm{PC}$ of the category $2 \mathrm{PCA}$ is $82 \%$. 
A)

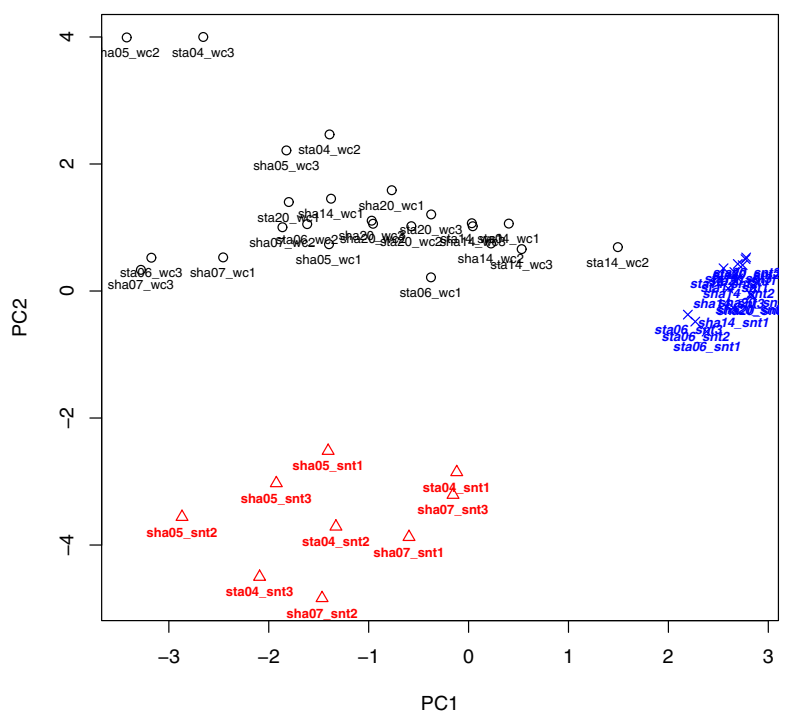

B)

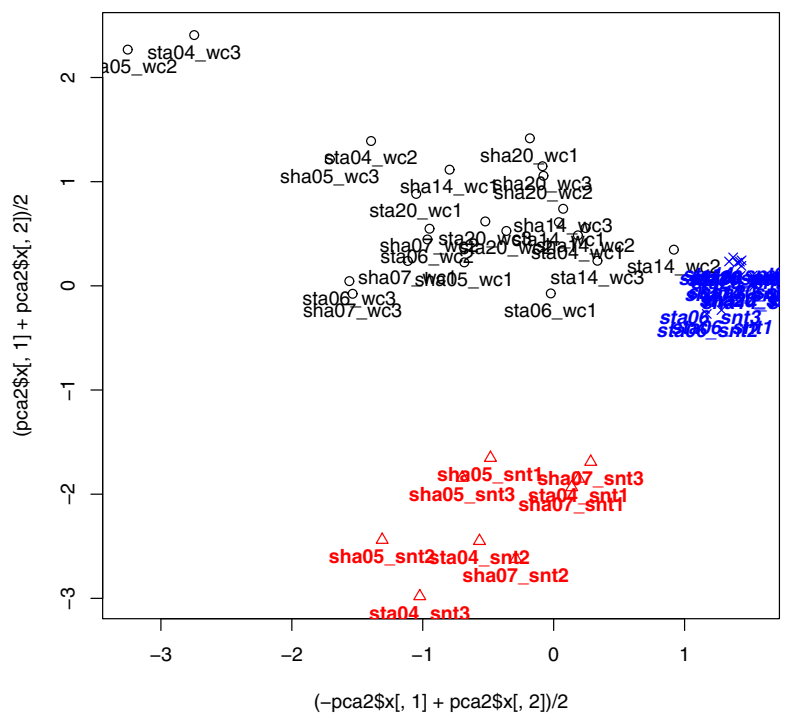

Fig. 2. A) Two dimensional embeddings of samples by Category 1 PCA, after the exclusion of $P^{\prime}$ proteins in Table 1 B) The same as A) but using only the selected $P "=30$ proteins shown in Table 2. Cumulative contribution up to the second PC of the category $2 \mathrm{PCA}$ is $67 \%$. 
The proteomes of $S$. pyogenes SF370, that grew under shaking or static culture condition, were clustered into three groups (Figures 1 and 2): the whole cellular proteome (all whole cellular experiments in Figures 1 and 2), the early phase extracellular proteome (sha05_snt, sha07_snt, and sta04_snt experiments in Figures 1 and 2), and the late phase extracellular proteome (sha14_snt, sha20_snt, sta06_snt, sta14_snt, and sta20_snt experiments in Figure 1] and 2), respectively. These results indicate that the proteomic phenotypes of $S$. pyogenes were divided into the two growth stages, the early growth phase that consists of the states at 5 and 7 hours under the shaking condition and the state at 4 hours under the static condition, and the late growth phase that consists of the states at the 14 and 20 hours under the shaking condition and the states at the 6, 14, and 20 hours under the static condition. It is suggested that the proteomic phenotype that grows under the static condition might rapidly grow from the early growth stage to the late growth stage compared with the shaking culture condition. Since the cell density $\left(O D_{660}\right)$ at 5 hour under the shaking condition and the cell density at 4 hour under the static condition are the same value $\left(O D_{660}=0.4\right)$ and the cell density at 7 hour under the shaking condition and the cell density at 6 hour under the static condition are the same value $\left(O D_{660}=0.8\right)$, the proteome is dependent upon the cellular fraction (whole cell or extracellular) or the time development rather than the culture condition.

\subsection{Biological Meanings of Representative Proteins}

In Tables 1 and 2, we have shown representative proteins for rounds one and two. Figures 3 and 4 show expressions of the below mentioned proteins among those.

In this study, there are four designed experimental groups characterized by the combination of two criteria: two fractions (the whole cellular component or the supernatant component) and two culture conditions (incubation with or without shaking). Several proteins are group-specific and are picked up by PCA. For example, peroxiredoxin reductase (SPy2079:AhpC), which is estimated to be involved in oxygen metabolism and hydrogen peroxide decomposition, is found in shaking culture condition rather than static condition. It seems reasonable that the amount of AhpC increases in shaking condition because the shaking condition induces the higher oxygen stress. On the other hand, twenty out of the fifty-three representative proteins picked up with PCA are ribosomal subunit proteins (the proteins underlined in Tables 1 and 2). This number is as many as a half of ribosomal proteins identified in this study, while a total number of ribosomal proteins annotated in SF370 genome is fifty-three. These twenty ribosomal proteins were picked up with PCA due to the abundance in the cellular fraction (not shown here). The reason why several ribosomal proteins were also found in extracellular fraction (as a typical example, see SPy0055:rplV in Fig. 3) is possibly because of the leakage during cell division (see below).

Besides, many virulence associated proteins, pyogenic exotoxin B (SpeB; SPy2039), pyogenic exotoxin C (SpeC; SPy0711), mitogenic factors (Mf; SPy2043, Mf2; SPy0712, and Mf3; SPy1436), and M protein (Emm; SPy2018), 

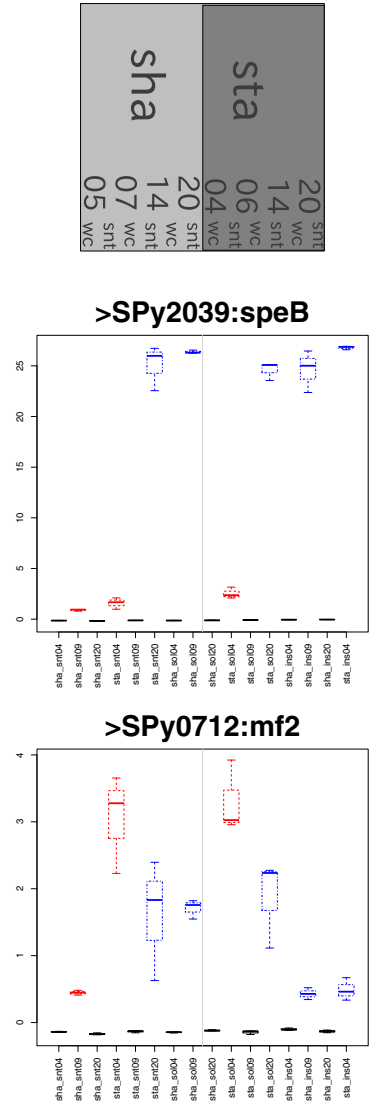

>SPy0273:fus

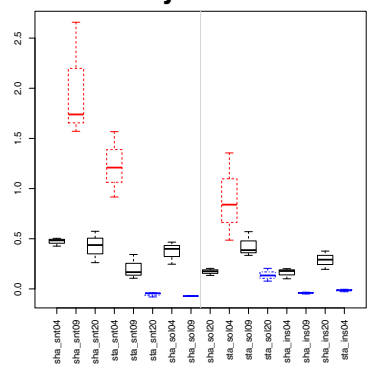

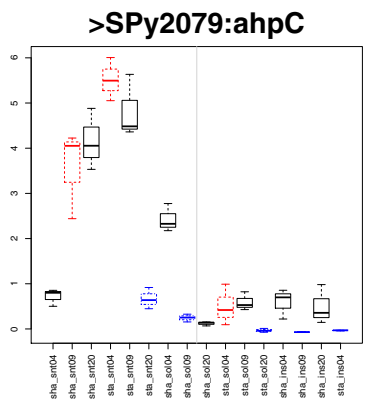
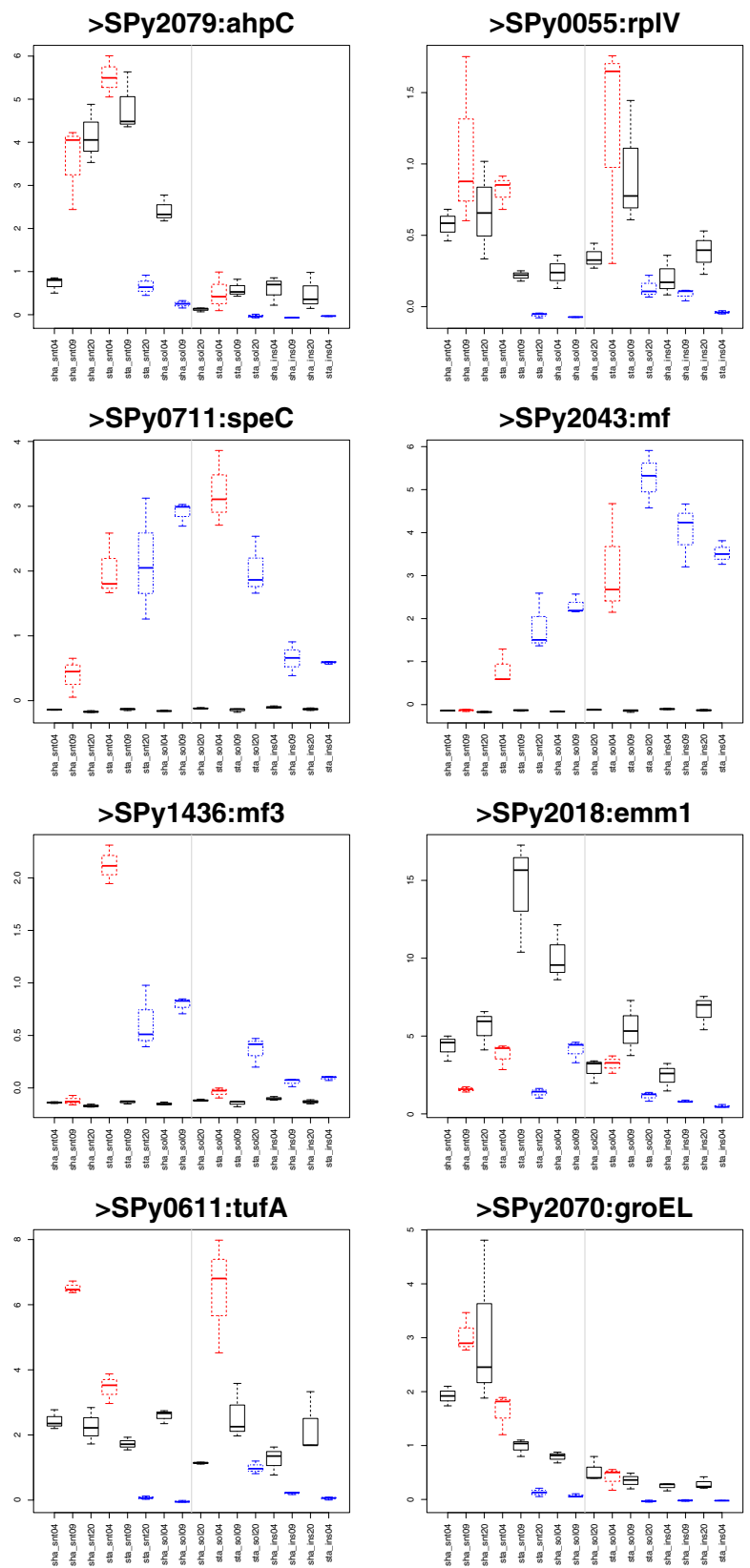

>SPy2070:groEL

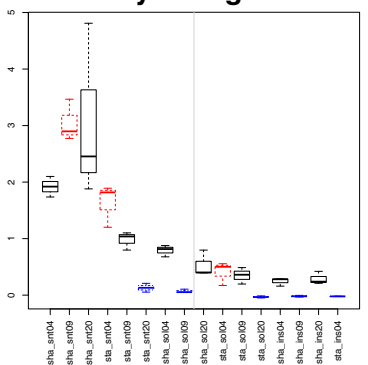

Fig. 3. Expression of representative proteins mentioned in the text. Colors and line types (black solid lines, red broken lines, and blue dotted broken lines) correspond to the colors in Figs. 1 and 2, The top-left panel: Schematic explanation of each panel. 

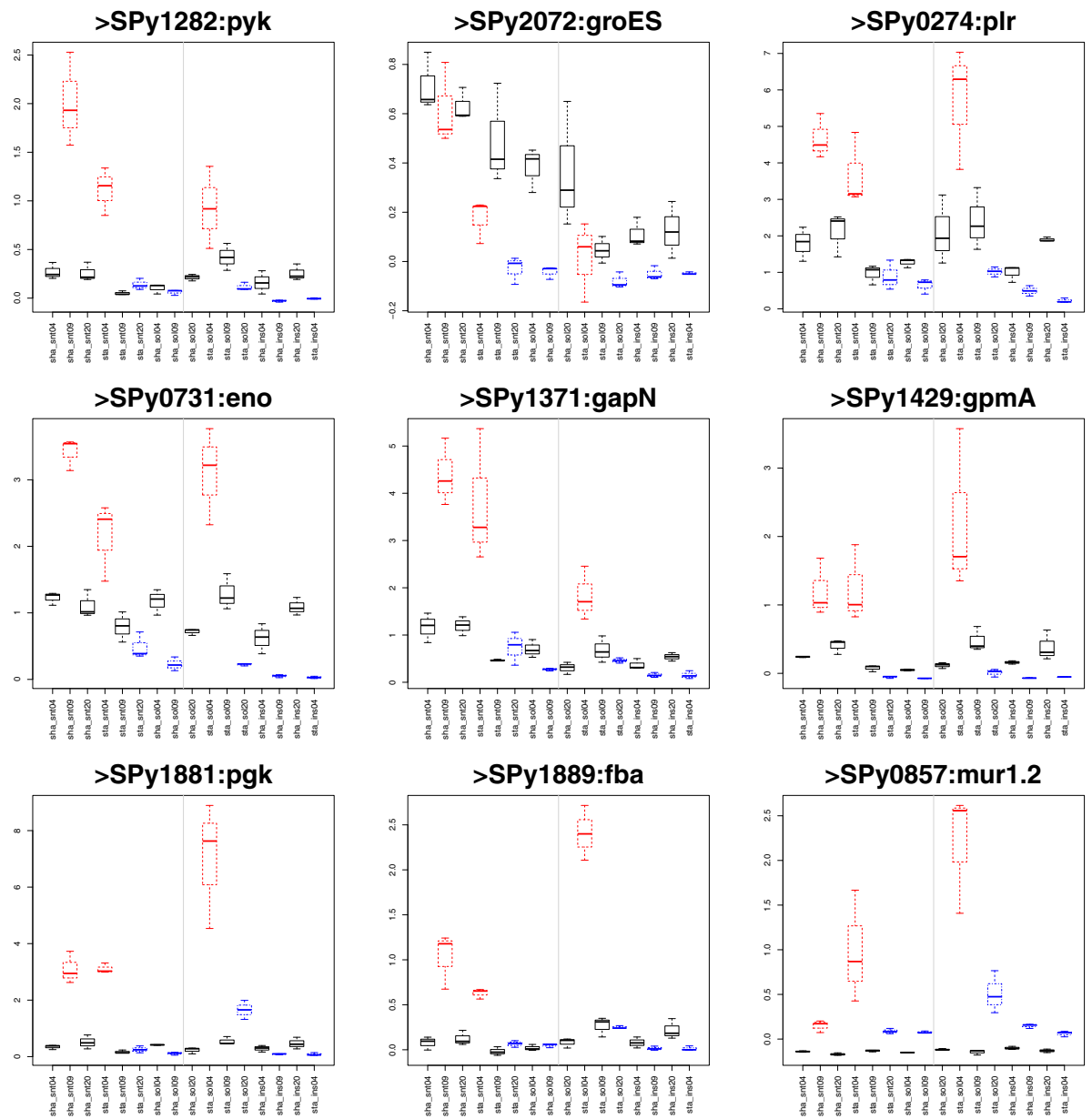

Fig. 4. Expression of representative proteins menthioned in the text. Notations are the same as Fig. 3 . 
are picked up by PCA analysis. These virulence-associated proteins have their own combination of the spatial and temporal distributions. SpeB increases monotonically in time, in both shaking and static culture condition. On the other hand, both Mf2 and SpeC increase under the shaking condition, but decrease under the static condition. The amount of both $\mathrm{M}$ protein and Mf increase and that of Mf3 decrease in shaking condition, although their amount keeps the constant value under the static incubation condition. The common distribution patterns are shared by the several abundant enzymes concerning the protein biosynthesis: such as an elongation factor EF-2 (Fus, SPy0273), an elongation factor Tu (TufA, SPy0611), a chaperonin (GroEL, SPy2070), and a co-chaperonin (GroES, SPy2072). The other common fashion of the protein distribution is also observed in enzymes involved in glycolysis: glyceraldehyde3-phosphate dehydrogenase (Plr, SPy0274), phosphopyruvate hydratase (Eno, SPy0731), pyruvate kinase (Pyk, SPy1282), NADP-dependent glyceraldehyde3-phosphate dehydrogenase (GapN, SPy1371), phosphoglyceromutase (GpmA, SPy1429), phosphoglycerate kinase (Pgk, SPy1881), and fructose-bisphosphate aldolase (Fba, SPy1889). Each protain is also observed by not small amount in the extracellular fraction at the early growth stage (sha05_snt, sha07_snt and sta04_snt, which are demonstrated by the red color in Fig. 3). They keep constant values throughout all sampling points in the whole cellular fraction. None of these proteins possessed signal sequence for secretion. Moreover, they are estimated to be intracellular enzymes such as the proteins involved in protein synthesis or glycolysis. It is confirmed the signal sequence-less proteins are always observed in the extracellular fraction of several bacterial species 6 6 ]. Most bacterial species that belong to firmicutes use autolytic enzymes, such as peptidoglycan hydrolase (Mur1.2, SPy0857), during the cell division processes [1138]. Mur1.2 is also observed in early growth stage. It is supposed that these proteins are leaked from cytoplasm during cell division, especially in early growth stage.

In conclusion, we have successfully selected biologically important proteins.

\subsection{Comparison with Transcriptome Analysis}

Although there are no transcriptomic analyses performed to investigate the difference between the shaking or static incubation conditions, there is a research where the transcriptome is compared between the stationary phase and the exponential phase [1. We also analysed these public domain data sets (see Materials and Methods) and tried to investigate if the gene coding the proteins picked up with PCA in this study show the significant difference between transcriptome between the static and exponential phases. In order to compare transcriptome between stationary and exponential phase, $P$-values, the rejection probability for the difference between the static and exponential phases, are attributed to transcruptome which corresponds to representative proteins. These $P$-values are compared with $P$-values for other proteins than representatives. Then $P$-values to depict the significant difference between two sets of $P$-values is obtained (Table 3). Both of $P$-values attributed to each of round one and two are mostly (21 out of 23 for round 1 and 23 out of 30 for round 2 ) less than $1 \times 10^{-3}$ (Wilcoxon 
Table 3. $P$-values (raw and $\mathrm{BH}$ corrected) attributed to representative proteins (Tables 1 and 2 ) obtained for transcriptome. Left:round one, midle and right: round two.

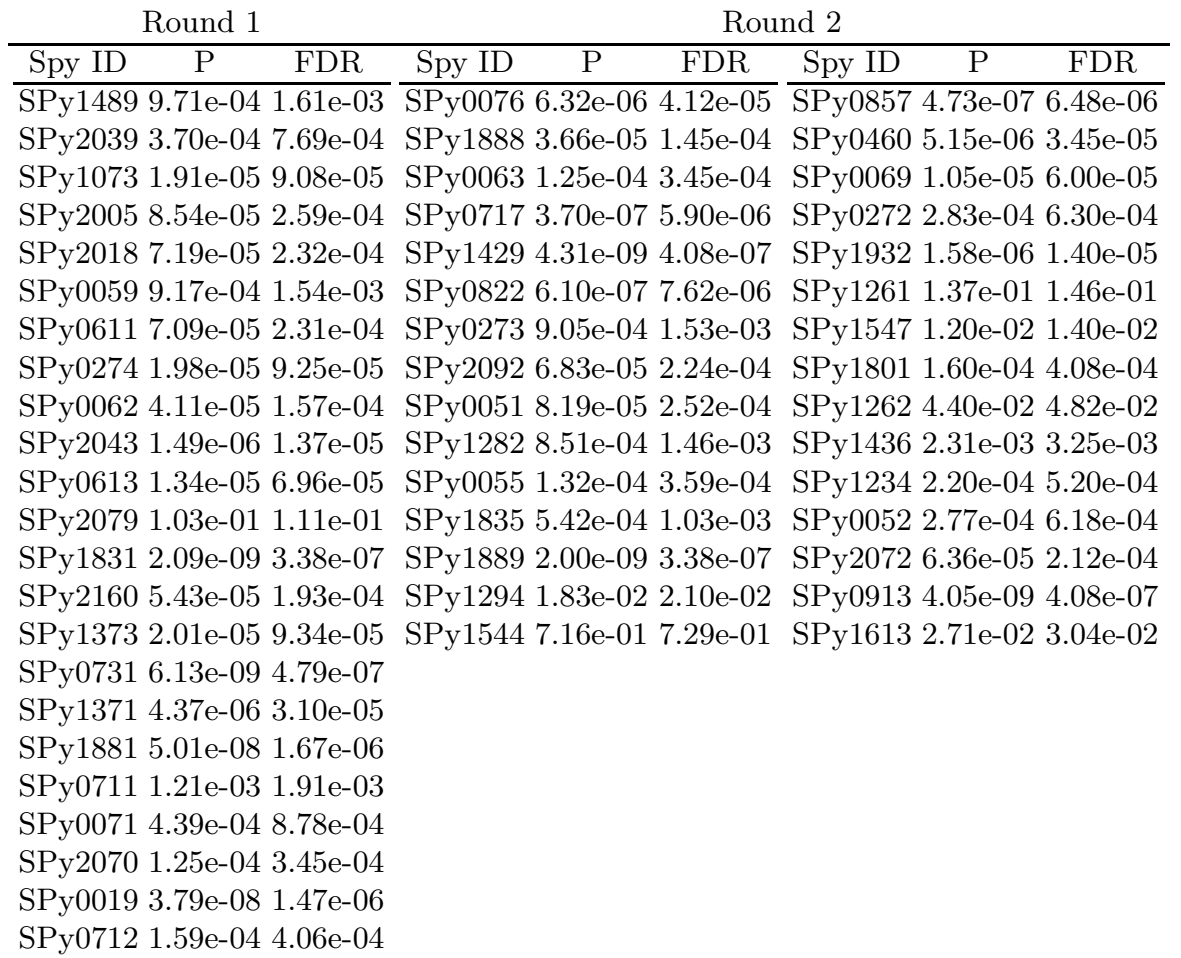

test). We have also computed FDR corrected $P$-values, they are still mostly $(22$ out of 23 for round 1 and 28 out of 30 for round 2) highly significant, i.e., less than $5 \times 10^{-2}$. This means, proteins whose expression differs between two culture conditions are also significantly different with each other in transcriptome levels between exponential-phase and stationary-phase. Since the difference between two culture conditions is supposed to be the difference of time scale as mentioned above, our selection of representative proteins based upon proteome data turns out to be coincident with transcriptome analysis.

\section{Conclusions}

In this paper, we have performed proteome analysis of Streptococcus pyogenes, under two distinct culture conditions; with or withour shaking. Representative proteins are selected by iterative applications of PCA in two ways. These proteins turn out to be biologically informative and their trasctiptome expression also differs significantly between early or late stages.

Acknowledgement. This work was supported by KAKENHI (23300357). 


\section{References}

1. Barnett, T.C., Bugrysheva, J.V., Scott, J.R.: Role of mRNA stability in growth phase regulation of gene expression in the group A streptococcus. J. Bacteriol. 189, 1866-1873 (2007)

2. Beyer-Sehlmeyer, G., Kreikemeyer, B., Hörster, A., Podbielski, A.: Analysis of the growth phase-associated transcriptome of streptococcus pyogenes. International Journal of Medical Microbiology 295(3), 161-177 (2005),

http://www.sciencedirect.com/science/article/pii/S1438422105000421

3. Blackman, S.A., Smith, T.J., Foster, S.J.: The role of autolysins during vegetative growth of Bacillus subtilis 168. Microbiology (Reading, Engl.) 144 ( pt. 1), 73-82 (1998)

4. Ferretti, J.J., McShan, W.M., Ajdic, D., Savic, D.J., Savic, G., Lyon, K., Primeaux, C., Sezate, S., Suvorov, A.N., Kenton, S., Lai, H.S., Lin, S.P., Qian, Y., Jia, H.G., Najar, F.Z., Ren, Q., Zhu, H., Song, L., White, J., Yuan, X., Clifton, S.W., Roe, B.A., McLaughlin, R.: Complete genome sequence of an M1 strain of Streptococcus pyogenes. Proc. Natl. Acad. Sci. U.S.A. 98, 4658-4663 (2001)

5. Ishihama, Y., Oda, Y., Tabata, T., Sato, T., Nagasu, T., Rappsilber, J., Mann, M.: Exponentially modified protein abundance index (emPAI) for estimation of absolute protein amount in proteomics by the number of sequenced peptides per protein. Mol. Cell Proteomics 4, 1265-1272 (2005)

6. Lei, B., Mackie, S., Lukomski, S., Musser, J.M.: Identification and immunogenicity of group A Streptococcus culture supernatant proteins. Infect. Immun. 68, 6807-6818 (2000)

7. Len, A.C., Cordwell, S.J., Harty, D.W., Jacques, N.A.: Cellular and extracellular proteome analysis of Streptococcus mutans grown in a chemostat. Proteomics 3, 627-646 (2003)

8. Mercier, C., Durrieu, C., Briandet, R., Domakova, E., Tremblay, J., Buist, G., Kulakauskas, S.: Positive role of peptidoglycan breaks in lactococcal biofilm formation. Mol. Microbiol. 46, 235-243 (2002)

9. Okamoto, A., Taguchi, Y.H.: Principal component analysis for bacterial proteomic analysis. IPSJ SIG Technical Report 2011-BIO-26, 1-6 (2011)

10. Okamoto, A., Yamada, K.: Proteome driven re-evaluation and functional annotation of the Streptococcus pyogenes SF370 genome. BMC Microbiol. 11, 249 (2011)

11. Oshida, T., Sugai, M., Komatsuzawa, H., Hong, Y.M., Suginaka, H., Tomasz, A.: A Staphylococcus aureus autolysin that has an N-acetylmuramoyl-L-alanine amidase domain and an endo-beta- $\mathrm{N}$-acetylglucosaminidase domain: cloning, sequence analysis, and characterization. Proc. Natl. Acad. Sci. U.S.A. 92, 285-289 (1995)

12. Rao, P.K., Li, Q.: Principal Component Analysis of Proteome Dynamics in Ironstarved Mycobacterium Tuberculosis. J. Proteomics Bioinform. 2, 19-31 (2009)

13. Shinoda, K., Tomita, M., Ishihama, Y.: emPAI Calc-for the estimation of protein abundance from large-scale identification data by liquid chromatography-tandem mass spectrometry. Bioinformatics 26, 576-577 (2010)

14. Taguchi, Y.H., Okamoto, A.: Principal component analysis for bacterial proteomic analysis. In: 2011 IEEE International Conference on Bioinformatics and Biomedicine Workshops, pp. 961-963 (2011) 